\title{
ON CURVATURE IN FINSLER GEOMETRY
}

\author{
BY \\ LOUIS AUSLANDER
}

Introduction. H. Busemann in [2] generalizes to Finsler geometry most of those global theorems in Riemannian geometry which relate the nonpositive curvature properties of the Riemannian metric to the topological properties of the manifold. This is carried out by means of his synthetic or axiomatic approach. We have approached the problem of generalizing theorems concerning positive as well as nonpositive curvature from the point of view of the theory of connections. We take as our starting point the connection for a Finsler manifold as calculated by Cartan in [3]. Using as a guide those theorems in Riemannian geometry which relate the position of conjugate points on a geodesic to the sectional curvature along the geodesic, we have given an analytic definition of sectional curvature which we believe is natural and which further permits us to generalize such theorems as: Myers' Theorem [5], which states that any complete Riemann manifold with positive mean curvature is compact; Synge's Theorem [8], which states that if an orientable even dimensional Riemann manifold is complete and has positive sectional curvature then it is simply connected; and that if $M$ is a simply connected, complete Riemann manifold that has nonpositive curvature, then it is homeomorphic to the Euclidean space of the same dimension.

We shall assume in presenting the details of this paper that the reader is familiar with E. Cartan's book [3] and with S. S. Chern's paper [4]. We wish to express our appreciation to Professor Chern without whose guidance and encouragement this work would not have been possible.

1. On the reduction of the group. Let $V$ be an $n$ dimensional vector space and let $D$ be the $n-1$ dimensional space of directions in $V . D$ may be defined as the equivalence classes of $V$, with the origin deleted, under the following equivalence relation: $v_{1}, v_{2} \in V$ will be said to be equivalent if there exists a constant $k$, greater than zero, such that $v_{1}=k v_{2}$. It is clear that $D$ may be considered topologically as an $n-1$ dimensional sphere. Now let $M$ be a differentiable manifold. Let $T$ be the tangent bundle to $M$ and let $S$ be the associated sphere bundle. Further, let $p: S \rightarrow M$ be the projection map. Then to each point $m \in M$ we may associate $V(m)$ and $D(m)$ as the fiber over the point $m$ in the bundles $T$ and $S$ respectively. We shall denote an element of $S$ by $(m, d)$ and an element of $T$ by $(m, v)$. We then have the well defined concept of the vector $v$ lying in the direction $d$ at $m$, provided $v$ is not the zero vector.

Presented to the Society, September 5, 1953; received by the editors July 28, 1954. 
Assume now that the manifold $M$ has a Finsler metric defined on it. Then to each point $(m, d)$ of $S$, we may associate a right-handed frame of vectors from $V(m)$. Let $\left(m, d ; e_{1}, \cdots, e_{n}\right)$ denote such an object. Let $P^{\prime}$ be the set of all such objects, where $(m, d)$ is any element of $S$ and $e_{1}, \cdots, e_{n}$ is any orthonormal frame at $(m, d)$. Then $P^{\prime}$ is a bundle over $S$ with fiber and group the $n$ dimensional orthogonal group. To show that this is the case, let $U$ be any coordinate neighborhood of $M$ with coordinates $x_{1}, \cdots, x_{n}$. Let $X_{1}=\partial / \partial x_{1}, \cdots, X_{n}=\partial / \partial x_{n}$ be the natural base for $V(m), m \in U$, induced by the coordinates $x_{1}, \cdots, x_{n}$. Then we may apply the Gram-Schmidt orthogonalization to $X_{1}, \cdots, X_{n}$. This will give a unique orthonormal frame of vectors in $V(m), e_{1}, \cdots, e_{n}$, for each point $(m, d) \in p^{-1}(U)$. We shall call $e_{1}, \cdots, e_{n}$ the associated orthonormal frame of vectors to $x_{1}, \cdots, x_{n}$ at $(m, d)$. Let $f_{1}, \cdots, f_{n}$ be any other orthonormal frame at $(m, d)$, then we associate with $f_{1}, \cdots, f_{n}$ the element of $O(n)$ which takes $e_{1}, \cdots, e_{n}$ into $f_{1}, \cdots, f_{n}$. This clearly gives $P^{\prime}$ locally the structure of a product space.

To define the full bundle structure we must define the coordinate functions. Let $U_{i}$ be an indexed family of coverings of $M$ by coordinate neighborhoods with indexing set $J$. Then $p^{-1}\left(U_{i}\right)=\bar{U}_{i}$ is an indexed family of coverings of $S$ with indexing set $J$. We wish to define a collection of mappings

$$
g_{i j}: \bar{U}_{i} \cap \bar{U}_{j} \rightarrow O(n)
$$

such that

$$
g_{k j}(x) g_{j i}(x)=g_{k i}(x) \text { in } \bar{U}_{i} \cap \bar{U}_{j} \cap \bar{U}_{k} .
$$

Let $m \in \bar{U}_{i} \cap \bar{U}_{j}$ and $U_{i}$ have coordinates $x_{1}, \cdots, x_{n}$ and associated righthanded frame $e_{1}, \cdots, e_{n}$ at $(m, d) \in \bar{U}_{i}$. Let $U_{j}$ have coordinates $y_{1}, \cdots, y_{n}$ and let $f_{1}, \cdots, f_{n}$ be the associated right-handed frame at $(m, d) \in \bar{U}_{j}$. Then since $f_{\alpha} \in V(m), \alpha, \beta=1, \cdots, n$, we have $f_{\alpha}=\sum a_{\alpha \beta} e_{\beta}$, where $\left(a_{\alpha \beta}\right) \in O(n)$. Let $g_{j i}(m, d)=\left(a_{\alpha \beta}\right)$. Clearly (1.2) holds. Then $P^{\prime}$ may be defined as follows: Let $J$ have the discrete topology and form

$$
S \times O(n) \times J .
$$

Define $(s, g, i)$ as equivalent to $\left(s^{\prime}, g^{\prime}, j\right)$ if

$$
s=s^{\prime} g_{i j}(g)=g^{\prime} \text {. }
$$

$P^{\prime}$ is then the equivalence classes of $S \times O(n) \times J$ under the above equivalence relation. Hence we have proven

TheOREM 1.1. Let $M$ be a Finsler manifold, then there exists a principal bundle $P^{\prime}$ over $S$ with fiber and group $O(n)$. Further, we may choose $p^{-1}\left(U_{i}\right)=\bar{U}_{i}$ as coordinate neighborhoods of this bundle structure.

The converse to Theorem 1.1 is also true; i.e., if over $S$ we can construct a principal bundle $P^{\prime}$ as above then $M$ may be given a Finsler metric by a 
process analogous to that one which is fully discussed in [7, paragraph 12] for Riemann metrics. We propose to show that the bundle $P^{\prime}$ can always be constructed over $S$.

Now we may define the action of the general linear group G.L. $(n, R)$ on $D$ by considering $D$ as equivalence classes of $V-\{0\}$. Let $\theta(n)$ be the group of matrices of the form

$$
\left(\begin{array}{ll}
Q & 0 \\
0 & Q
\end{array}\right)
$$

where $Q \in O(n)$ and where $O$ is the $(n, n)$ zero matrix. Clearly $\theta(n)$ is isomorphic to $O(n)$ and $\theta(n)$ may be considered as a subgroup of G.L. $(2 n, R)$.

Lemma 1.1. Let $M$ be a differentiable manifold, $T$ its tangent bundle, and let $T^{\prime}$ be the tangent bundle to the differentiable manifold $T$. Then the structure group, $G$, of $T^{\prime}$ over $T$ may be reduced to $\theta(n)$.

It can be shown that $G$ has matrix representation

$$
\left(\begin{array}{ll}
A & B \\
0 & A
\end{array}\right)
$$

where $A \in \mathrm{G}$.L. $(n, R)$ and $B$ is any $(n, n)$ matrix over the real field. Then $G / \theta(n)$ is homeomorphic to the topological product of G.L. $(n, R) / O(n)$ and the Euclidean space of dimension $n^{2}$, and Steenrod proves in $[7$, p. 57] that G.L. $(n, R) / O(n)$ is solid. Hence, since there exists a cross section in the associated bundle of $T^{\prime}$ with fiber $G / O(n)$, the group of the bundle $T^{\prime}$ can be reduced to $\theta(n)$. (See Corollary 12.6, p. 56 of [7].)

As in Lemma 1.1, let $T$ be the tangent bundle over a differentiable manifold $M$. Now consider the associated bundle to $T$ with fiber $D$. This exists because we have defined the action of G.L. $(n, R)$, on $D$. This is exactly the bundle $S$.

Since we have in Lemma 1.1 reduced the group of the bundle $T^{\prime}$ over $T$ to the group $\theta(n)$, and we have a natural isomorphism between $\theta(n)$ and $O(n)$, we may construct the associated bundle to $T^{\prime}$ with fiber and group $O(n)$. Call this bundle $B$. We wish to show that $B$ induces the bundle $P^{\prime}$ over $S$.

All we have to show is that the group $G$ can be considered as the structure group of $S$. Now let $\left(u^{* 1}, \cdots, u^{* n}\right)$ and $\left(u^{1}, \cdots, u^{n}\right)$ be two coordinate neighborhoods in $M$. Then

$$
\begin{aligned}
& u^{* i}=u^{* i}\left(u^{1}, \cdots, u^{n}\right), \\
& y^{* i}=\sum \partial u^{* i} / \partial u^{i} y^{i},
\end{aligned} \quad i, j=1, \cdots, n,
$$

are the transformation equations for $T$, where $y^{* i}=d u^{* i}$ and $y^{i}=d u^{i}$, because $\left(u^{1}, \cdots, u^{n} ; y^{1}, \cdots, y^{n}\right)$ and $\left(u^{* 1}, \cdots, u^{* n}, y^{* 1}, \cdots, y^{*_{n}}\right)$ are local co- 
ordinate systems for $T$. Now these equations preserve the equivalence relations of Definition (1.1). Hence we have defined the action of $G$ on $S$. Now exactly the same argument as the one given in Lemma 1.1 proves

Lemma 1.2. Over the bundle $S$ we may construct a principal bundle $P^{\prime}$ with fiber and group $O(n)$ and coordinate neighborhoods $p^{-1}(U)$ where $U$ is a coordinate neighborhood on $M$.

Let $Z$ be the space of equivalence classes of $V-\{0\}$ under the equivalence relation: $v_{1}$ is equivalent to $v_{2}$ if there exists a constant $k$ such that $v_{1}=k v_{2}$. Then we can carry through the entire discussion as before replacing $D$ by $Z$.

Now Steenrod has proven that the space $Y$, consisting of symmetric positive definite matrices, is solid $[7$, p. 58]. Hence

THEOREM 1.2. Every differentiable manifold has a non-Riemannian symmetric Finsler metric.

2. Existence of geodesic coordinates. Consider a Finsler space $M$ and a geodesic segment $G$, which we shall denote parametrically as $g(t), 0 \leqq t \leqq a$, $a>0$. Assume that $g\left(t_{1}\right)=g\left(t_{2}\right)$ if and only if $t_{1}=t_{2}$. Let $\left(g_{0}, e_{n} ; e_{1}, \cdots, e_{n}\right)$ denote a fixed element of $P^{\prime}$ over $g_{0}=g(0)$. Since $G$ is a geodesic we may use parallel displacement to define a path in $P^{\prime}$ covering $G$. Now consider the geodesic $G_{\alpha}$ in the direction $e_{\alpha}$ at $g_{0}$. If we let $t$ denote the arc length of $G$ and let $s_{\alpha}$ denote the arc length on $G_{\alpha}$, then the Jacobian of the system of equations

$$
\begin{aligned}
t & =t\left(x_{1}, \cdots, x_{n}\right), \\
s_{\alpha} & =s_{\alpha}\left(x_{1}, \cdots, x_{n}\right), \quad \alpha=1, \cdots, n-1,
\end{aligned}
$$

where $\left(x_{1}, \cdots, x_{n}\right)$ is any coordinate system at $g_{0}$, equals the determinant of the components of the vectors $e_{i}, i=1, \cdots, n$, in the $\left(x_{1}, \cdots, x_{n}\right)$ coordinate system and hence is not zero.

Definition 2.1. The coordinate system defined above by $\left(s_{\alpha}, t\right)$ about $g_{0}$ is called a geodesic coordinate system.

Let $g\left(t_{1}\right)$ and $g\left(t_{2}\right)$ be two points on $G$. Then we may consider $G$ as a metric space with the distance between $g\left(t_{1}\right)$ and $g\left(t_{2}\right)$ defined as $\left|t_{2}-t_{1}\right|$. Let $\rho\left(g\left(t_{1}\right)\right.$, $\left.g\left(t_{2}\right)\right)$ be the Finsler distance between these two points. Then, since a geodesic locally minimizes the distance between two points of $M$, for each $g \in G$ there exists an open neighborhood of $g, U(g) \subset G$, such that for any two points $g_{1}=g\left(t_{1}\right)$ and $g_{2}=g\left(t_{2}\right)$ in $U(g), \rho\left(g_{1}, g_{2}\right)=\left|t_{2}-t_{1}\right|$. If to each $g \in G$ we associate such an open set, we get an open covering of the compact metric space $G$. Let $e>0$ be the Lebesgue number of this covering.

Now for each point $g \in G$, let $W(g)$ be an open set in $M$ such that the geodesic coordinate svstem defined before has nonvanishing Jacobian. Let $d>0$ be the Lebesgue number of this covering. For $g \in G$, let $O(g)$ be an open 
interval on $G$ with center $g$ and diameter equal to the minimum of $e$ and $d$. Define the function $\xi(g)$ by the equation

$$
\xi(g)=\text { g.l.b. } \rho\left(g, g^{\prime}\right),
$$

where $g^{\prime} \in G-O(g)$. Clearly $\xi(g)$ is a continuous function of $g$. Define $\xi=$ g.l.b. $\in_{g} \xi(\mathrm{g})$.

\section{Lemma 2.1. $\xi>0$.}

Proof. Assume the lemma is false; since $G$ is compact this is equivalent to the assertion that $\xi\left(g_{0}\right)=0$ for some $g_{0}$. Then there exists a sequence of points $g_{i}=g\left(t_{i}\right)$ such that $\rho\left(g_{0}, g_{i}\right)$ converges to zero as $i$ goes to infinity, but $t_{i}$ must be such that $\left|t_{i}-t_{0}\right|>e$. Hence $t_{i}$ cannot converge to $t_{0}$. Since $G$ is compact we must have $g\left(t^{\prime}\right)=g(t)$ for $t \neq t^{\prime}$. This contradicts our definition of $G$.

Note that $\min (e, d) \geqq \xi$.

THEOREM 2.1. If we restrict the diameter of $O(g)$ to be less than $\xi / 2$, then the geodesic coordinate system may be defined in $\mathrm{U}_{0} \in{ }_{G} O(g)$.

Proof. Assume the theorem is false; i.e., the point $m$ in $\cup O(g)$ has coordinates $\left(t^{\prime}, s^{\prime}\right)$ and $(t, s)$, where $\left(t^{\prime}, s^{\prime}\right) \neq(t, s), s=\left(s_{1}, \cdots, s_{n-1}\right)$, and $s^{\prime}=\left(s_{1}^{\prime}\right.$, $\left.\cdots, s_{n-1}^{\prime}\right)$. Then $\left|t-t^{\prime}\right| \geqq \xi$. By the triangle inequality we have

$$
\rho\left(g(t), g\left(t^{\prime}\right)\right) \leqq \rho(g(t), m)+\rho\left(m, g\left(t^{\prime}\right)\right) .
$$

By hypothesis $\rho(g(t), m)$ and $\rho\left(m, g\left(t^{\prime}\right)\right)$ must each be less than $\xi / 2$. Therefore $\rho\left(t, t^{\prime}\right)<\xi$. Hence, by definition of $\xi,\left|t-t^{\prime}\right|=\rho\left(t, t^{\prime}\right)$. Hence $\left|t-t^{\prime}\right|$ $>\left|t-t^{\prime}\right|$. This is clearly a contradiction and the theorem follows.

3. On the bundle $P$. We have seen that the principal bundle $P^{\prime}$ may be considered as the set of elements of the form $\left(m, d ; e_{1}, \cdots, e_{n}\right)$, where $e_{1}, \cdots, e_{n}$ is an orthonormal frame of vectors in $V(m)$ at $(m, d)$. Let $P$ be the subset of elements of $P^{\prime}$ with the property that $e_{n}$ lies in the direction of $d$. We shall denote elements of $P$ by $\left(m ; e_{1}, \cdots, e_{n}\right)$. Then it is clear that $P$ is a bundle over $S$ with fiber $O(n-1)$ and group $O(n-1)$. Further $P$ is a principal bundle over $M$ with fiber and group $O(n)$.

We shall henceforth use the bundle $P$ rather than $P^{\prime}$ in our discussion.

Professor Chern has calculated the equations of structure for a Finsler metric [4] and they are given as follows, when all forms are considered over the bundle $P$.

$$
\begin{aligned}
d w_{i} & =\sum w_{j} \wedge w_{j i}+\pi_{i} \\
d w_{i j} & =\sum w_{i k} \wedge w_{k j}+\pi_{i j}
\end{aligned}
$$

where

$$
w_{i j}+w_{j i}=0
$$




$$
\pi_{i j}=\sum \frac{1}{2} Q_{i j \alpha \beta} w_{\alpha n} \wedge w_{\beta n}+\sum P_{i j k \alpha} w_{k} \wedge w_{\alpha n}+\frac{1}{2} \sum R_{i j k l} w_{k} \wedge w_{l}
$$

where summation is over all indices on the right-hand side of (3.4).

Now let $\bar{U} \subset S$ be a local cross section over $U \subset M$ such that the projection map is a differentiable homeomorphism. The injection map $i: \bar{U} \rightarrow S$ induces a bundle $\bar{P}$ over $\bar{U}$ with fiber and group $O(n)$. Let $\bar{R}$ be the associated bundle with the positive definite symmetric matrices as fiber. Then there exists a natural map $i: \bar{P} \rightarrow P$. Hence we may pull the forms $w_{i}, w_{i j}$ back to $\bar{P}$ by means of the induced mapping $\bar{i}^{*}$. Let $\bar{i}^{*}(w)=\bar{w}$. Now the cross section $f: S \rightarrow R$ induces a cross section $\bar{f}: \bar{U} \rightarrow \bar{R}$. This cross section clearly defines a Riemann metric on $\bar{U}$. This is called the induced Riemann metric.

ThEOREM 3.1. The forms $\bar{w}_{i}$ define the induced Riemann metric by

$$
d s^{2}=\sum_{i=1}^{n}\left(\bar{w}_{i}\right)^{2}
$$

and the forms $\bar{w}_{i j}$ define the affine connection associated with the induced Riemann metric.

The proof of this theorem follows from the formula

$$
d f^{*} w=f^{*} d w, \quad f^{*}\left(w \wedge w^{\prime}\right)=f^{*} w \wedge f^{*} w^{\prime} \quad \text { and } \quad \bar{w}_{j i}+\bar{w}_{i j}=0 .
$$

We shall now study more explicitly the definition of this induced Riemann metric for certain cross sections. Let $G$ be a geodesic in $M$ and let $\bar{U}$ contain $\bar{G}$. Consider a geodesic coordinate neighborhood about $G$, with coordinates $x_{1}, \cdots, x_{n}$, where $t=x_{n}, s_{\alpha}=x_{\alpha}, \alpha=1, \cdots, n-1$. Now the cross section into $P$ which defines the Finsler metric over $\bar{G}$ merely picks out $n$ covectors in $V^{*}(m), m \in G$, i.e., $d x_{1}, \cdots, d x_{n}$. Now $\bar{U}$ and $p(\bar{U})$ may be coordinatized by the same coordinates $x_{1}, \cdots, x_{n}$ and hence there is a natural isomorphism between $V^{*}(\bar{m})$ and $V^{*}(p(\bar{m}))$. Hence the cross section restricted to $\bar{U}$ also picks $n$ vectors out of $V^{*}(\bar{m})$; i.e., $d x_{1}, \cdots, d x_{n}$. These are therefore an orthonormal frame at $\bar{m} \in \bar{G}$. Hence we have

THEOREM 3.2. $\bar{w}_{i}=w_{i}$ along $\bar{G}$.

Now it is well known that we may consider our Finsler metric as a local metric which assigns arc length to certain paths in $S$; namely, those paths which can be obtained from paths in the base space by associating with each point of the curve its tangent direction. This process we shall call lifting. If $C$ is a curve in $M$, we shall denote the lifted curve by $\bar{C}$.

In light of this discussion, we have the arc length along $\bar{G}$ in the Finsler metric and any induced Riemannian cross section containing $\bar{G}$ is exactly the same. 
THEOREM 3.3. Let $\bar{U}$ be any cross section containing a lifted geodesic $\bar{G}$. Consider $\bar{U}$ as a Riemann manifold with its induced Riemann metric. Then $\bar{G}$ is a geodesic in $\bar{U}$.

Proof. We have along $\bar{G}$ in $S$ that $w_{\alpha}=0, \alpha=1, \cdots, n-1$ and $w_{n \alpha}=0$, [1]. Hence $\bar{w}_{\alpha}=0, \bar{w}_{n \alpha}=0$ along $\bar{G}$ in $\bar{U}$. Hence $\bar{G}$ is a geodesic in $\bar{U}$.

Let the equations of structure on $\bar{U}$ be given by

$$
\begin{aligned}
d \bar{w}_{i} & =\sum \bar{w}_{j} \wedge \bar{w}_{j i}, \\
d \bar{w}_{i j} & =\sum \bar{w}_{i k} \wedge \bar{w}_{k j}+\sum R_{i j k l} \bar{w}_{k} \wedge \bar{w}_{l} .
\end{aligned}
$$

Then along $\bar{G}$, if we assume $\bar{w}_{n}$ is the arc length along $\bar{G}$, we have

Theorem 3.4. $\bar{R}_{n \alpha n \beta}=\sum P_{n \alpha n \gamma} A_{n \gamma \beta}+R_{n \alpha n \beta}$, along $\bar{G}$, where $P_{n \alpha n \gamma}$ and $R_{n \alpha n \beta}$ are given by equation (3.4) and $A_{n \gamma \beta}$ are functions depending on the cross section $\bar{U}$ chosen.

Proof. Along $\bar{G}$ we have

$$
\begin{aligned}
d \bar{w}_{i j}=\sum \bar{w}_{i k} \wedge \bar{w}_{k j}+ & \frac{1}{2} \sum Q_{i j \alpha \beta} \bar{w}_{\alpha n} \wedge \bar{w}_{\beta n}+\sum P_{i j k \alpha} w_{k} \wedge \bar{w}_{\alpha \beta} \\
+ & \frac{1}{2} \sum R_{i j k l} w_{k} \wedge w_{l}
\end{aligned}
$$

where $Q_{n j \alpha \beta}=0$. But along $\bar{G}$ we have $\left\langle\bar{w}_{n \alpha}, d x_{n}\right\rangle=0$. Hence $\bar{w}_{n \alpha}=A_{n \alpha \beta} w_{\beta}$, along $\bar{G}$. Substitution proves the theorem.

4. Curvature. Let $(m, d) \in S$. Let $G$ be a geodesic in $M$ with tangent directions $d$ at $m$. Let $\bar{U}$ be any local cross section in $S$ containing $\bar{G}$. By a local cross section we mean a cross section in the bundle $S$ restricted to some open set in $M$. Then at $(m, d)$ we may define sectional curvature for any 2-dimensional plane $E \subset V(m)$ containing the direction $d$ relative to $\bar{U}$ as follows:

Under the homeomorphism between $M$ and $\bar{M}$ let $E$ go into $\bar{E} \subset V(\bar{m})$. Then define the sectional curvature in the induced Riemann metric as the sectional curvature for the plane $E$ relative to the cross section $\bar{U}$ at $m$. Denote this by $R(\bar{U}, m, d, v)$, where $d$ and $v$ span $E$.

THEOREM 4.1. Let $\bar{U}_{1}$ and $\bar{U}_{2}$ satisfy the above definition and assume that they are tangent at $\bar{m}$ in $S$. Then $R\left(\bar{U}_{1}, m, d, v\right)=R\left(\bar{U}_{2}, m, d, v\right)$.

Proof.

$$
\begin{aligned}
& R\left(\bar{U}_{1}, \bar{m}, \bar{E}\right)=\left(P_{n \alpha n \gamma} A_{n \gamma \beta}+R_{n \alpha n \beta}\right) X_{\alpha} X_{\beta}, \\
& R\left(\bar{U}_{2}, \bar{m}, \bar{E}\right)=\left(P_{n \alpha n \gamma} B_{n \gamma \beta}+R_{n \alpha n \beta}\right) X_{\alpha} X_{\beta},
\end{aligned}
$$

where $X_{\alpha}$ are the components of a unit vector, $X$, perpendicular to $w_{n}$. This follows from Theorem 3.4 and the definition of sectional curvature. Hence 
since $P_{n \alpha n \gamma}$ and $R_{n \alpha n \beta}$ depend only on the point $(m, d)$ and not the cross section $\bar{U}$ chosen, all we have to show is that when $\bar{U}_{1}$ and $\bar{U}_{2}$ are tangent at $(m, d), A_{n \beta \gamma}=B_{n \beta \gamma}$. But by definition

$$
\bar{w}_{n \alpha}=A_{n \alpha \beta} w_{\beta}, \quad \bar{w}_{n \alpha}=B_{n \alpha \beta} w_{\beta}
$$

for $\bar{U}_{1}$ and $\bar{U}_{2}$ respectively. But the induced mappings depend only on the tangent plane at $(m, d)$ in $S$. This proves the theorem.

Definition 4.1. $R(\bar{U}, m, d, v)=R(F, m, d, v)$, where $F$ is the hypertangent plane to $\bar{U}$ at $(m, d)$, is called the sectional curvature at $(m, d)$ and $E$ relative to the plane $F$.

Definition 4.2. Let $M(\bar{U}, m, d)$ be the mean curvature on the point $(m, d)$ in the direction $d$. Then $M(\bar{U}, m, d)$ is called the mean curvature at $m$ relative to the direction $d$.

It is clear that these definitions specialize to the usual ones in Riemannian geometry.

THEOREM 4.2. Let $G$ be a geodesic segment which is not a relative minimum. Then $\bar{G}$ is not a relative minimum for some cross section $\bar{U}$.

THEOREM 4.3. Let $G$ be a geodesic and let $\bar{G}$ not be a relative minimum for some cross section $\bar{U}$. Then $G$ is not a relative minimum in $M$.

Proof.

$$
\delta^{2} I=\left[\delta \phi_{n}+\sum \phi_{\alpha} \phi_{\alpha n}\right]_{0}^{1}+\int_{0}^{1}\left(\phi_{\alpha n} \phi_{\alpha n}+P_{n \alpha n \beta} \phi_{\alpha} \phi_{\beta n}+R_{n \alpha n \beta} \phi_{\alpha} \phi_{\beta}\right) w_{n}
$$

is the second variation along a geodesic in Finsler geometry [1]. Now

$$
\delta^{2} I=\left[\delta \phi_{n}+\sum \phi_{\alpha} \bar{\phi}_{\alpha n}\right]_{0}^{1}+\int_{0}^{1} \sum\left(\bar{\phi}_{\alpha n} \bar{\phi}_{\alpha n}+P_{n \alpha n \beta} \phi_{\alpha} \bar{\phi}_{\beta n}+R_{n \alpha n \beta} \phi_{\alpha} \phi_{\beta}\right) w_{n}
$$

is the variation along $\bar{G}$ in $\bar{U}$. Now $\phi_{\alpha n}=\bar{\phi}_{\alpha n}$ if the variation is contained in $\bar{U}$. This proves the two theorems.

A Finsler manifold $M$ is said to have positive mean curvature (sectional curvature) greater than or equal to $e^{2}$ if the mean curvature (sectional curvature) of all cross sections is greater than or equal to $e^{2}$ at all points and for all directions (all plane elements).

Since $A_{n \alpha \beta}$ are arbitrary, the assumption of positive sectional curvature is equivalent to the assumption that the following two conditions hold at every point and for all directions:

(a) $P_{i j k l}$ is the zero tensor.

(b) $R_{i j k l} X^{i} Y^{i} X^{k} Y^{l}>0$, where $X$ and $Y$ are perpendicular unit vectors relative to the direction $X$.

Theorem 4.4. A complete $n$ dimensional Finsler manifold $M$ whose mean 
curvature is greater than or equal to $e^{2}$ is compact and has diameter less than or equal to $\pi / e$.

Proof. Since $M$ is complete, any two points may be connected by a geodesic, $G$, whose length realizes the distance between the two points. We shall prove that if the diameter of $M$ is greater than $\pi / e$, then the distance between the two points is less than the length of $G$. This will contradict the definition of $G$. To do this, we shall show that the second variation is negative along $G$.

Let $\bar{U}$ be any cross section containing $\bar{G}$. We now shall calculate the second variation of $\bar{G}$ in $\bar{U}$ and show that it is negative.

Let $x_{1}, \cdots, x_{n}$ be a geodesic coordinate system in $\bar{U}$ about $\bar{G}$. Let $\left(\eta_{1}, \cdots, \eta_{n-1}\right)$ be the variation vector which is zero in all but the $\alpha$ place where it is $\sin \pi s / L$, where $s$ is the arc length parameter of $\bar{G}$ and $L$ equals total length of $\bar{G}$. Computing $\delta^{2} I$ by the usual formulas for the second variation in Riemannian geometry [6], we have

$$
\delta^{2} I\left(\eta_{\alpha}\right)=\int_{a}^{b}\left\{\frac{\pi^{2}}{L^{2}} \cos ^{2} \frac{\pi s}{L}-\sin ^{2} \frac{\pi s}{L} \bar{R}_{n \alpha n \alpha}\right\} d s
$$

where $\bar{R}_{n \alpha n \alpha}$ has been computed in Theorem 3.4. Now

$$
\int_{a}^{b} \cos ^{2} \frac{\pi s}{L} d s=\int_{a}^{b} \sin ^{2} \frac{\pi s}{L} d s
$$

Thus

$$
\delta^{2} I\left(\eta_{\alpha}\right)=\int_{a}^{b} \sin ^{2} \frac{\pi s}{L}\left(\frac{\pi^{2}}{L^{2}}-\bar{R}_{n \alpha n \alpha}\right) d s .
$$

Now summing over all $\alpha$, we get

$$
\sum \delta^{2} I\left(\eta_{\alpha}\right)=\int_{a}^{b} \sin ^{2} \frac{\pi s}{L}\left[(n-1) \frac{\pi^{2}}{L^{2}}-(n-1) M\left(\bar{U}, m, e_{n}\right)\right] d s .
$$

Assume $L>\pi / 3$. Then $\pi^{2} / L^{2}<e^{2}<M\left(\bar{U}, m, e_{n}\right)$. Thus $\sum \delta^{2} I\left(\eta_{\alpha}\right)<0$. Hence $\delta^{2} I\left(\eta_{\alpha}\right)<0$, for at least one $\alpha$. Hence $\bar{G}$ is not a relative minimum. But by Theorem 4.3 this implies that $G$ is not a relative minimum. This is the desired contradiction.

TheOREM 4.5. A complete n-dimensional Finsler manifold whose mean curvature is positive has finite fundamental group.

If this theorem is false then the universal covering space of $M, \tilde{M}$, is noncompact. But $M$ and $\tilde{M}$ are locally isometric and mean curvature is a local property. Hence the assumption that the fundamental group is not finite leads to a contradiction of Theorem 4.4.

Consider the diagram: 


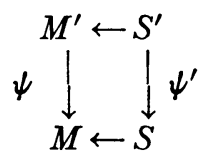

where $M^{\prime}$ is the universal covering of $M$; we assume it has a Finsler metric induced by the mapping $\psi^{-1}$, where $S$ and $S^{\prime}$ are the sphere bundles over $M$ and $M^{\prime}$ respectively and where the mapping $\psi^{\prime}$ is defined by

$$
\psi^{\prime}\left(V_{i} \times S^{n-1}\right)=\psi\left(V_{i}\right) \times S^{n-1}
$$

in terms of local coordinate neighborhoods in $S^{\prime}$ and $S$ respectively. It is then clear that $S^{\prime}$ is a covering space of $S$. Further, let $\bar{M} \subset S^{\prime}$ be a local cross section with its induced Riemann metric. Then $\psi^{\prime}\left(\bar{M}^{\prime}\right)$ is a local cross section over $M, \bar{M}^{\prime}$ is a covering space over $\psi^{\prime}\left(\bar{M}^{\prime}\right)=\bar{M}$, and the two induced Riemann metrics on $\bar{M}^{\prime}$ are identical.

Theorem 4.6. A complete orientable even dimensional Finsler manifold $M$ of positive sectional curvature is simply connected.

Assume the theorem is false. It is a well known theorem that in every free homotopy class of $M$, there exists a shortest geodesic. Since $M$ is not simply connected there exists a nontrivial free homotopy class and hence a nontrivial shortest geodesic $G$.

Let $G^{\prime}$ cover $G$ in $M^{\prime}$ with end points $a$ and $b$. Then $G^{\prime}$ is a geodesic with respect to the Finsler metric on $M^{\prime}$ and $\psi(a)=\psi(b)$.

LEMMA 4.1. $G^{\prime}$ is not a relative minimum in any local cross section $\bar{U}^{\prime}$ containing it.

Proof. Let $\bar{a}^{\prime}$ be the initial point of $\bar{G}^{\prime}$ and $\bar{b}^{\prime}$ be its end point. Let $V\left(\bar{a}^{\prime}\right)$ and $V\left(\bar{b}^{\prime}\right)$ be the tangent spaces in $\bar{U}^{\prime}$ at $\bar{a}^{\prime}$ and $\bar{b}^{\prime}$, respectively, which are perpendicular to the geodesic $\bar{G}^{\prime}$. Since $\bar{U}^{\prime}$ is a covering space of $\bar{U}$ and $\psi^{\prime}\left(\bar{a}^{\prime}\right)=\psi^{\prime}\left(\bar{b}^{\prime}\right)$, we may use the covering map to induce a mapping $f: V\left(\bar{a}^{\prime}\right)$ $\rightarrow V\left(b^{\prime}\right)$ such that $f$ is orthogonal and orientation preserving. Therefore $f$ is a rotation.

Let $v$ be a normal vector to $\bar{G}^{\prime}$, in $\bar{U}^{\prime}$ at $\bar{a}^{\prime}$. Consider the parallel displacement of $v$ along $\bar{G}^{\prime}$. We have

$$
\delta^{2} I=\int-R\left(\bar{U}^{\prime}, m, e_{n}, v\right) d s .
$$

Since $R\left(\bar{U}, m, e_{n}, v\right)>0$ we have

$$
\delta^{2} I(\eta)<0
$$

Hence $\bar{G}^{\prime}$ is not a relative minimum.

Proof of Theorem 4.6. Let $v^{\prime}$ be the vector parallel to $v$ at $b^{\prime}$. Let $v^{*}=f^{-1}\left(v^{\prime}\right)$. Consider the map $F$ of $V\left(\bar{a}^{\prime}\right)$ onto itself defined by $F(v)=v^{*}$. Since the mani- 
fold is even dimensional, the unit sphere in the space $V\left(\bar{a}^{\prime}\right)$ is also even dimensional. Hence the mapping $F$ has a fixed point. Call it $v_{0}$. Hence we get a strip about $\bar{G}^{\prime}$ which projects under $\psi^{\prime}$ into a closed strip in $\bar{U}$. But in this closed strip the curve $\bar{G}$ can be shortened as a closed curve in the same free homotopy class as $\bar{G}$. From Theorem 4.3 we then see that in $M$ there exists a geodesic in the same free homotopy class as $G$ which is shorter than $G$. This contradicts the definition of $G$ and thereby proves our theorem.

A manifold $M$ is said to have nonpositive curvature if all of its sectional curvatures for all cross sections are less than or equal to zero.

Theorem 4.7. Let $M$ be a complete, simply connected analytic Finsler manifold of nonpositive curvature, then it is homeomorphic to the Euclidean space of the same dimension.

Proof. By [9] we see we may choose polar coordinates about a point $m \in M$. Since we have nonpositive curvature the geodesics through $m$ contain no points conjugate to $m$. Hence this coordinate system is valid all over $M$ and this proves the theorem.

\section{BIBLIOGRAPHY}

1. L. Auslander, On the use of forms in the variational calculations, to appear in the Pacific Journal of Mathematics.

2. H. Busemann, Spaces with non-positive curvature, Acta Math. vol. 80 (1948) pp. 259-310.

3. E. Cartan, Les espaces de Finsler, Actualités Scientifiques et Industrielles, no. 79, Paris, Hermann, 1934.

4. S. S. Chern, Euclidean connection for Finsler spaces, Proc. Nat. Acad. Sci. U. S. A. vol. 29 (1943) pp. 38-43.

5. S. B. Myers, Riemannian manifolds with positive mean curvature, Duke Math. J. vol. 8 (1941) pp. 401-404.

6. I. J. Schoenberg, Some applications of the calculus of variations to Riemannian geometry, Ann of Math. vol. 33 (1932) pp. 485-495.

7. N. Steenrod, The topology of fibre bundles, Princeton University Press, 1951.

8. J. L. Synge, On the connectivity of spaces of positive curvature, The Quarterly Journal of Mathematics vol. 7 (1936) pp. 316-320.

9. J. H. C. Whitehead, On the covering of a complete space by geodesics through a point, Ann. of Math. vol. 36 (1935) pp. 679-704.

YALE UNIVERSity,

New Haven, Conn. 